\title{
Subcutaneous fat necrosis in neonates after therapeutic hypothermia - report of two cases
}

\author{
Dawid Szpecht, Alina Bagnosz-Magnuszewska, Marta Szymankiewicz, Janusz Gadzinowski
}

Department of Neonatology, Poznan University of Medical Sciences, Poznan, Poland

Adv Dermatol Allergol 2016; XXXIII (2): 152-154

DOI: 10.5114/ada.2016.59164

Subcutaneous fat necrosis (SCFN) is a rare, transient and self-limiting complication of birth asphyxia [1]. It is characterized by painless nodules developing on the back, limbs and buttocks, which move freely over muscle and bone. The overlying skin may be of normal color or with erythematous discoloration. These lesions disappear spontaneously without scarring, but there may be systemic complications associated with SCFN. The most serious potential complication is hypercalcemia, which carries with it serious risks to the child's health and life [1].

Both neonates diagnosed with SCFN following therapeutic hypothermia were born with indicators of serious birth asphyxia in the Clinical Hospital of Gynecology and Obstetrics at Poznan University of Medical Sciences.

A female newborn weighing $4340 \mathrm{~g}$ was delivered by cesarean section (indication: fetal life-threatening symptoms) at 37 weeks of pregnancy complicated by type C diabetes and Hashimoto's thyroid deficiency. She was born with an Apgar score of 0 at $1 \mathrm{~min}$, and then 0 , 0, 1 and 4 at 3, 5, 10 and 15 min, respectively. Umbilical cord $\mathrm{pH}$ (artery and vein) was measured immediately after birth at $6.71(\mathrm{BE}-21.2 \mathrm{mEq} / \mathrm{l})$ and $6.78(-21.0 \mathrm{mEq} / \mathrm{l})$. The child was resuscitated at birth, with heart action returning at $10 \mathrm{~min}$. Artificial ventilation (initially by the synchronized intermittent mechanical ventilation (SIMV) method and then with non-invasive ventilation support) in the Neonatal Intensive Care Unit (NICU) was continued until day 10 of her life. Due to clinical symptoms of hypoxic ischemic encephalopathy whole-body cooling was initiated at $4 \mathrm{~h}$, and continued for the next 3 days of life.

In the first cranial ultrasound scan performed within the initial $24 \mathrm{~h}$ of the infant's life, no hypoxic-ischemic areas were detected. Abnormal values of pulsatility index (PI) of 3.4, resistive index (RI) of 0.93, end-diastolic velocity (EDV) of $3.5 \mathrm{~cm} / \mathrm{s}$ and peak systolic velocity (PSV) of $50.5 \mathrm{~cm} / \mathrm{s}$ were detected in the middle cerebral artery. Subsequent cranial ultrasound scans done at weekly intervals revealed minor ischemic areas around cerebral ventricles, also confirmed by cranial magnetic resonance imaging in week 5 of the patient's life.

As of day 32 of her life, the infant presented reddish discoloration of the skin and subcutaneous nodules located primarily on her back and along the posterior side of her arms. Skin lesions were accompanied by the following abnormal results of laboratory tests: hypercalcemia (peak level of $\mathrm{Ca}^{2+}$ at day 23: $2.9 \mathrm{mmol} / \mathrm{l}$; reference range: 2.2-2.5 mmol/l), hypertriglyceridemia (peak triglyceride level at day 23: $217 \mathrm{mg} / \mathrm{dl}$; reference range: below $150 \mathrm{mg} / \mathrm{dl}$ ). The newborn was put on a low-calcium, low-vitamin $\mathrm{D}_{3}$ diet, and diuretics were administered. As a result, skin lesions were gradually resolving, and the $\mathrm{Ca}^{2+}$ levels and lipid metabolism were normalized. The baby was discharged from hospital at day 42 of her life, with a good general health status. In a neurological examination muscle hypertonia and excessive tendon reflexes were detected.

A female full-term neonate weighing $5070 \mathrm{~g}$ was delivered by cesarean section in week 39 of pregnancy because of fetal life-threatening symptoms. The pregnancy was complicated by maternal $G 2$ diabetes. The child was born with an Apgar score of 5, 2, 2, 5 and 6 at 1, 3, 5, 10 and $15 \mathrm{~min}$, respectively. Umbilical cord $\mathrm{pH}$ (artery and vein) was measured immediately after birth at 6.79 (BE $-18.9 \mathrm{mEq} / \mathrm{l})$ and $6.98(-15.0 \mathrm{mEq} / \mathrm{l})$. After resuscitation the neonate was transferred to the NICU, where artificial ventilation (initially by the SIMV method and then with non-invasive ventilation support) was applied until day 5 . Because of signs of serious intrauterine hypoxia, therapeutic hypothermia (COOL-CAP) was applied. She showed symptoms of pulmonary hypertension, hypotension, periodical clonic-tonic seizures, and bleeding from the urinary and respiratory tract resulting from hemorrhagic diathesis. The levels of liver enzymes were considerably elevated from the first $24 \mathrm{~h}$ of her life: alanine

Address for correspondence: Dawid Szpecht MD, Department of Neonatology, Poznan University of Medical Sciences, 33 Polna St, 60-535 Poznan, Poland, phone: +48 502145 181, e-mail: dawid.szpecht@poczta.fm Received: 25.03.2015, accepted: 28.05.2015. 
transaminase (ALT) $1131.40 \mathrm{U} / \mathrm{l}$, aspartate transaminase (AST) $3175.70 \mathrm{U} / \mathrm{l}$. Similarly, the level of troponin was elevated and it provided evidence of damage to the cardiac muscle $(0.798 \mathrm{ng} / \mathrm{ml}$; normal range up to $0.1 \mathrm{ng} / \mathrm{ml})$. The markers of renal activity, initially within their normal ranges, were following a rising trend over several consecutive 24-hour periods of the baby's life, peaking on day 9 (urea $224.0 \mathrm{mg} / \mathrm{dl}$, creatinine $1.60 \mathrm{mg} / \mathrm{dl}$ ).

In the cranial ultrasound scan performed on day 3 , after therapeutic cooling of the newborn ended, no deviations from the norm were found, whereas another scan on day 6 of life showed abnormal, blurred echogenicity and, after 2 weeks, considerable increased heterogeneity in periventricular areas - an indication of possible leukomalacia. In neurologic assessment decreased muscle tone along the head-torso axis and increased tone in upper and lower limbs were detected. Physiotherapy treatment (Vojta method) and stimulation of the sucking reflex were applied during the period of hospitalization at the intensive care unit.

In her second month of life, physical examination revealed multiple subcutaneous painless nodules, ca. $0.5-1.0 \mathrm{~cm}$ in diameter, located on the thorax, the back, the extensor surface of arms, the external side of thighs and the buttocks. The clinical symptoms were accompanied by abnormal calcium and lipid metabolism. When the subcutaneous lesions emerged, total plasma calcium level was $3.10 \mathrm{mmol} / \mathrm{l}$, and it peaked at day 35 of life at $4.0 \mathrm{mmol} / \mathrm{l}$; triglyceride level was $386 \mathrm{mg} / \mathrm{dl}$. The infant was put on a low-calcium/low-vitamin $D_{3}$ infant formula, and steroid therapy (hydrocortisone in a dose of $1 \mathrm{mg} /$ $\mathrm{kg}$ ) was administered. No spectacular improvement in skin condition was achieved after 2 weeks of therapy (the lesions were still present at discharge from hospital). Total plasma calcium level at that point was $2.70 \mathrm{mmol} / \mathrm{l}$ and triglyceride level was $508.9 \mathrm{mg} / \mathrm{dl}$. The patient was discharged from hospital at day 48 of her life, with recommendations that the low-calcium/low-vitamin $D_{3}$ diet should continue and follow-up visits in the Endocrinology Department be arranged.

Subcutaneous fat necrosis is a serious metabolic disorder. Usually the condition is self-limiting and resolves spontaneously. It occurs in full-term neonates who were subject to perinatal stress. The literature points to several risk factors leading to the development of SCFN, including birth asphyxia, hypothermia and meconium aspiration syndrome. Subcutaneous fat necrosis is more common in infants born to mothers with gestational diabetes, arterial hypertension, preeclampsia and eclampsia. In the cases of the 2 infants described in this paper these risk factors were present: both infants were born after a pregnancy complicated by diabetes, suffered asphyxia at birth and were, because of that, treated with therapeutic hypothermia. It is also believed that factors predisposing to the development of SCFN include anemia and thrombocytopenia [1]. The results of hypo- thermia treatment for neonates with hypoxic ischemic encephalopathy, presented by the National Institute of Child Health and Human Development, revealed dermatological complications in the form of SCFN in 3.9\% (4 of 102) of newborn infants included in a randomized study [2]. In the pathogenesis of SCFN an important role is played by peripheral perfusion disturbances which in consequence lead to crystallization of free fatty acids and subcutaneous fat necrosis. Varan et al. [3] point out that both anemia and polycythemia in the perinatal period are conducive to peripheral hypoperfusion and increase the risk of SCFN. Neither of the 2 infants described in this paper had anemia or polycythemia. Other factors postulated as significant contributors to the pathogenesis of SCFN are the immaturity of the enzymatic system involved in desaturation of free fatty acids and local, congenital or acquired defects of fatty tissue [3]. Clinical symptoms of SCFN usually develop after the first $72 \mathrm{~h}$ of the newborn's life, in the form of discolored skin patches and subcutaneous nodules a few millimeters to a few centimeters in size, and they most often affect the skin on the arms, legs and on the back [1, 3, 4]. The neonates treated in the Department of Neonatology in Poznan presented skin symptoms of SCFN primarily on the back and on the arms, and the lesions developed only at the beginning of the second month of their lives. A number of metabolic disorders, such as hypertriglyceridemia, hypercalcemia and hypoglycemia, as well as hematologic disorders, such as anemia and thrombocytopenia, are observed in the course of SCFN. It seems that an essential role in the pathogenesis of hypoglycemia is played by birth asphyxia and related multiple organ dysfunction. Metabolic disorders more often found in infants born to mothers with diabetes [5-7]. Mothers of both neonates described in this paper had diabetes. Neither of the infants had hypoglycemia; however, starting from the first hour of their lives, both of them were totally parenterally fed. Hypercalcemia is a rare yet life-threatening complication of SCFN. Calcium ions are released from damaged adipocytes and the production of $1,25-\mathrm{OH}-\mathrm{D}_{3}$ in infants with SCFN takes place in an uncontrolled manner, also outside kidneys. Moreover, increased calcium absorption in the intestine is observed [5, 7-9]. In our patients non-symptomatic hypercalcemia was found. Treatment for hypercalcemia involves a low-calcium diet, diuretics and corticosteroids. The therapy for hypercalcemia in our patients with SCFN included modified milk (low in $\mathrm{Ca}^{2+}$ and vitamin $D_{3}$ ) and diuretics. Since this alone did not produce normalization of $\mathrm{Ca}^{2+}$ levels (case 2 ), corticosteroids were administered (hydrocortisone in a dose of $1 \mathrm{mg} / \mathrm{kg}$ ), which led to an improvement in laboratory test results. The therapy, under the supervision of an endocrinologist, continued after the infants were discharged from hospital. It is indicated in the literature that, in justified cases, treatment modalities for SCFN may include calcitonin and bisphosphonates [10]. Subcutaneous fat 
necrosis is diagnosed on the basis of its clinical picture. The histopathologic test of biopsy material taken from a nodule will show foci of necrosis and calcification in the fat tissue and infiltrates of mononucleated cells, macrophages and multinucleated giant cells $[1,4,6]$. Because the skin lesions in our patients did not cause any doubts, we did not proceed with the invasive procedure of a histopathology test of the skin.

To sum up, SCFN may represent a life-threatening complication of therapeutic neonatal hypothermia. It is advised that newborn infants treated with hypothermia for perinatal asphyxia should be regularly checked for the presentation of skin symptoms. Blood calcium levels in patients with subcutaneous fat necrosis should be monitored once a week over a period of six months after the disappearance of skin lesions typical for SCFN [10].

\section{Conflict of interest}

The authors declare no conflict of interest.

\section{References}

1. Hogeling M, Meddles K, Berk DR, et al. Extensive subcutaneous fat necrosis of the newborn associated with therapeutic hypothermia. Pediatr Dermatol 2012; 29: 59-63.

2. Shankaran S, Laptook AR, Ehrenkranz RA, et al. National Institute of Child Health and Human Development Neonatal Research Network. Whole-body hypothermia for neonates with hypoxic-ischemic encephalopathy. N Engl J Med 2005; 353: 1574-84.

3. Varan B, Gürakan B, Ozbek N, et al. Subcutaneous fat necrosis of the newborn associated with anemia. Pediatr Dermatol 1999; 16: 381-3.

4. Mahe E, Girszyn N, Hadj-Rabia S, et al. Subcutaneous fat necrosis of the newborn: a systemic evaluation of the risk factors, clinical manifestations, complications and outcome of 16 children. Br J Dermatol 2007; 156: 709-15.

5. Shumer DE, Thaker V, Taylor GA, et al. Severe hypercalcemia due to subcutaneous fat necrosis: presentation, management and complications. Arch Dis Child Fetal Neonatal Ed 2014; 99: 419-21.

6. Tran JT, Sheth AP. Complications of subcutaneous fat necrosis of the newborn: a case report and review of the literature. Pediatr Dermatol 2003; 20: 257-61.

7. Bonnemains L, Rouleau S, Sing G, et al. Severe neonatal hypercalcemia caused by subcutaneous fat necrosis without any apparent cutaneous lesion. Eur J Pediatr 2008; 167: 1459-61.

8. Borgia F, De Pasquale L, Cacace C, et al. Subcutaneous fat necrosis of the newborn: be aware of hypercalcaemia. J Paediatr Child Health 2006; 42: 316-8.

9. Dudink J, Walther FJ, Beekman RP. Subcutaneous fat necrosis of the newborn: hypercalcaemia with hepatic and atrial myocardial calcification. Arch Dis Child Fetal Neonatal Ed 2003; 88: 343-5.

10. Aucharaz KS, Baker EL, Millman GC, et al. Treatment in hypercalcemia in subcutaneous fat necrosis is controversial. Horm Res 2007; 68: 31. 